\title{
Development of the Variable Emittance Thermal Suite for the Space Technology 5 Microsatellite
}

\author{
Donya M. Douglas', Theodore Swanson', Robert Osiander ${ }^{2}$, John Champion ${ }^{2}$, \\ Ann Garrison Darrin ${ }^{2}$, William Biter ${ }^{3}$, and Prasanna Chandrasekhar \\ 'Thermal Engineering Branch, MS 545, NASA Goddard Space Flight Center, Greenbelt MD 20771 \\ 'The Johns Hopkins University Applied Physics Lab, Laurel MD 20723 \\ ${ }^{3}$ Sensortex, Inc. 515 Schoolhouse Road, Kennett Square, PA 19348 \\ ${ }^{4}$ Ashwin-Ushas Corporation, Inc., 500 James St., Unit 7, Lakewood, NJ 08701, USA \\ (301)286-6952,Donya.M.Douglas@gsfc.nasa.gov
}

\begin{abstract}
The advent of very small satellites, such as nano and microsatellites, logically leads to a requirement for smaller thermal control subsystems. In addition, the thermal control needs of the smaller spacecraft/instrument may well be different from more traditional situations. For example, power for traditional heaters may be very limited or unavailable, mass allocations may be severely limited, and fleets of nano/microsatellites will require a generic thermal design as the cost of unique designs will be prohibitive. Some applications may require significantly increased power levels while others may require extremely low heat loss for extended periods. Small spacecraft will have low thermal capacitance thus subjecting them to large temperature swings when either the heat generation rate changes or the thermal sink temperature changes. This situation, combined with the need for tighter temperature control, will present a challenging situation during transient operation. The use of "off-the-shelf" commercial spacecraft buses for science instruments will also present challenges. Older thermal technology, such as heaters, thermostats, and heat pipes, will almost certainly not be sufficient to meet the requirements of these new spacecraftinstruments. They are generally too heavy, not scalable to very small sizes, and may consume inordinate amounts of power. Hence there is a strong driver to develop new technology to meet these emerging needs. Variable emittance coatings offer an exciting alternative to traditional control methodologies and are one of the technologies that will be flown on Space Technology 5, a mission of three microsatellites designed to validate "enabling" technologies. Several studies have identified variable emittance coatings as applicable to a wide range of spacecraft, and to potentially offer substantial savings in mass and/or power over traditional approaches. This paper discusses the development of the variable emittance thermal suite for ST-5. More specifically, it provides a description of and the infusion and validation plans for the variable emittance coatings.
\end{abstract}

\section{INTRODUCTION}

NASA's New Millennium Program (NMP) endeavors to test new spacecraft technologies for future space and Earth Science missions who's primary objective will be to make multiple simultaneous measurements of the harsh space environment near the boundary of Earth's protective magnetic field known as the magnetosphere. Current satellites weigh hundreds to thousands of grams and use hundreds of watts of heater power. Future missions could involve constellations of $20+$ miniature satellites weighing 5 to $10 \mathrm{~kg}$ each that use a fraction of the power associated with larger spacecraft. In addition, these satellites could have perigees of $3 \mathrm{Re}$ and apogees of $12 \mathrm{Re}$ to $40 \mathrm{Re}$, resulting in orbital periods of 1.2 to 5.8 days and eclipses as long as 8 hours. The goal of NMP is to validate new technologies that will enable the reduction of weight, size, and cost of future Earth Science missions. As such, Space Technology 5 (ST-5), the fourth deep space mission in the NMP, will validate seven "enabling" technologies, which are listed in Table 1. Each of the technologies were selected by NMP and fall into two categories, spacecraft miniaturization and constellation technologies. In addition to the seven official technologies, ST-5 will fly a miniature digital sun sensor and science grade magnetometer. All of these components will be packaged into a spacecraft weighing $25 \mathrm{~kg}$ or less. ST-5, which is scheduled to launch in late 2003 , will fly as a secondary payload on a single expendable launch vehicle (Table 2, Mission Characteristics). The three satellites will be deployed 
successively into a highly elliptical orbit with a final perigee altitude of at least $200 \mathrm{~km}$ and an apogee altitude of no higher than $38,000 \mathrm{~km}$. Given the goal of NMP, ST-5's main objectives are to:

(1) demonstrate the ability to achieve accurate research quality scientific measurements using this class of spacecraft

(2) design, develop, and operate multiple spacecraft to act as a single constellation rather than as individual elements

(3) design, develop, integrate, test and operate a full-service $20 \mathrm{~kg}$ class spacecraft through the use of multiple new technologies (Table 2 NMP Technologies)

TABLE 1. NMP Technologies.

\begin{tabular}{llc}
\hline Technology & \multicolumn{1}{c}{ Type } & Provider \\
\hline Multifunctional Structures & Spacecraft Miniaturization & Lockheed-Martin \\
Variable Emittance Thermal Suite & Spacecraft Miniaturization & GSFC \\
Micro-thrusters & Spacecraft Miniaturization & Marotta Scientific Controls \\
Ultra Low Power (1/4 V Logic) & Spacecraft Miniaturization & GSFC \\
$\begin{array}{l}\text { Miniature Communications } \\
\text { Components }\end{array}$ & Spacecraft Miniaturization & \\
$\begin{array}{l}\text { Constellation Communication } \\
\text { and Navigation Transceiver }\end{array}$ & Constellation Technologies & AeroAstro \\
$\begin{array}{l}\text { Software Tools for } \\
\text { Autonomous Ground Operations }\end{array}$ & Constellation Technologies & JPL \\
\hline
\end{tabular}

TABLE 2. Mission Characteristics.

\begin{tabular}{|c|c|}
\hline Number of Satellites & 3 \\
\hline $\begin{array}{l}\text { Mission Orbit } \\
\text { Perigee } \\
\text { Apogee } \\
\text { Inclination } \\
\text { Period } \\
\text { Eclipse Duration }\end{array}$ & $\begin{array}{l}200 \mathrm{~km} \text { (minimum) } \\
38,000 \mathrm{~km} \text { (maximum) } \\
15 \text { to } 28.5^{\circ} \\
10.5 \mathrm{hrs} \\
35 \text { minutes (perigee eclipse), } 113 \text { minutes (apogee eclipse) }\end{array}$ \\
\hline Mission Lifetime & 3 months minimum, 6 month goal \\
\hline Spin Rate & 20 rpm (fully deployed) \\
\hline Launch System & Atlas V, III or Delta IV \\
\hline Orbit Adjust & Cold Gas System \\
\hline Mass & $<25 \mathrm{~kg}$ \\
\hline Size & $50.8 \mathrm{~cm}$ Diameter $\times 28.3 \mathrm{~cm} \mathrm{High}$ \\
\hline Power (Available to load) & $\sim 18.823$ Watts EOL \\
\hline Data & Max of $100 \mathrm{kbps}$ \\
\hline $\begin{array}{l}\text { Attitude Control } \\
\text { Control: } \\
\text { Knowledge: }\end{array}$ & $\begin{array}{l}\text { Spin Stabilized, with spin axis perpendicular to the sun } \\
\pm 5^{\circ} \text { (spin axis pointing control, } 3 \sigma \text { ) } \\
\pm 1^{\circ} \text { (spin axis pointing knowledge, } 3 \sigma \text { ) }\end{array}$ \\
\hline $\begin{array}{l}\text { Communications } \\
\text { Routine } \\
\text { Command }\end{array}$ & $\begin{array}{l}X-B \text { and } \\
X-B \text { and }\end{array}$ \\
\hline $\begin{array}{l}\text { Telemetry } \\
\text { Clock Accuracy: }\end{array}$ & Knowledge to $5 \mathrm{msec}$ \\
\hline
\end{tabular}




\section{BACKGROUND}

A variety of trends in scientific spacecraft i.e. greatly reduced size and mass, short design/build cycles, restricted resources (power, command, control, etc.), are driving the need for new thermal control technologies. Design of small spacecraft such as those for the ST-5 mission, are particularly challenging. Spacecraft in this very small size range, 10 to $20 \mathrm{~kg}$, will require smaller thermal control subsystems (Douglas; 2001). Specifically, small spacecraft will have low thermal capacitance thus subjecting them to large temperature swings when either the heat generation rate or the thermal sink temperature changes. The ST-S Constellation, for example, has a requirement to passively maintain thermal control through a one hour eclipse. This will be challenging without some means of modulating the heat rejection rate. Older thermal technologies, such as heaters, thermostats, or heat pipes, will almost certainly not be suitable to meet the requirements of these new spacecraft. They are generally too heavy, not scaleable to very small sizes, and may consume inordinate amounts of power. One of the technologies selected for validation on ST-5 is a suite of specialized thermal control coatings which can adjust their emittance based upon an on-board passive controller or from ground control.

Variable emittance thermal control coatings have been under development at GSFC since the mid-1990's. These coatings change the effective infrared emissivity of a thermal control surface to allow the radiative heat transfer rate to be modulated upon command. This permits adaptive or "smart" thermal control of spacecraft by varying effective emissivity of surfaces in response to a low voltage signal. Essentially an "electronic louver", this idea would have generic applicability to all spacecraft and be especially useful for truly micro and nanospacecraft.

It appears possible to develop such "electronic louvers" through at least three different types of technologies:

1) Electrochromic technology

2) Electrostatic technology

3) Micro Electro-Mechanical Systems (MEMS) technology

The following section provides a description of each of the three technologies.

\section{DESCRIPTION OF TECHNOLOGIES}

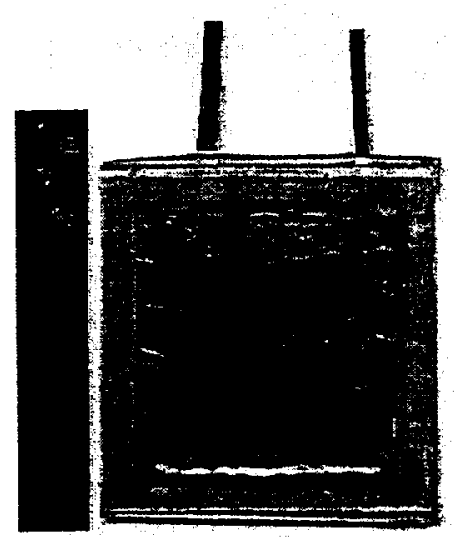

FIGURE 1. Reflectance Mode Electrochromic Device.

The first technology, the reflectance mode electrochromic devices, is being developed by Ashwin-Ushas located in Lakewood, New Jersey. Shown in Figure 1, the technology is based on a conductive polymer that utilizes electrochromic principles to modulate surface emissivity. The electrochromic device is based on a 2 -electrode design with a solid electrolyte, yielding a unique, entirely solid state device that is thin, flexible and lightweight. Device areas range from 1 to $225 \mathrm{~cm}^{2}$ with a thickness of 1 to $2 \mathrm{~mm}$ and an estimated weight of $160 \mathrm{mg} / \mathrm{cm}^{2}(1.6$ $\mathrm{kg} / \mathrm{m}^{2}$ ). The emittance modulation is achieved using electrochromic devices whose reflectance can be tuned over a broad wavelength range ( 0.4 to 2.5 microns in the Visible-NIR and 2.5 to 45 microns in the IR). The 
electrochromic process is based on reversible redox of Conducting Polymers ( $\mathrm{CPs}$ ) causing a change in the light absorption by these CPs. In the Visible-NIR, this occurs through optical absorptions of chromophores. In the IR, it occurs due to absorptions of conductive, bipolaron states in the $\mathrm{CP}$. These absorptions modulate the reflectance provided by an underlying Au coating on the flexible, microporous substrate, this being the global basis of the Visible/IR modulation. When a small bias voltage (typically $+/ .1 \mathrm{VDC}$ ) is applied, the ions shuttle to one side or the other, thus changing the effective emissivity of the surface. This change in the low infrared range produces an optimal $\Delta \varepsilon$ of 0.6 . The last set of emittance testing yielded $\Delta \varepsilon$ values of approximately 0.35 , emittance switching times from 1 to 7 seconds, and a cyclability greater than $10^{4}$ cycles.

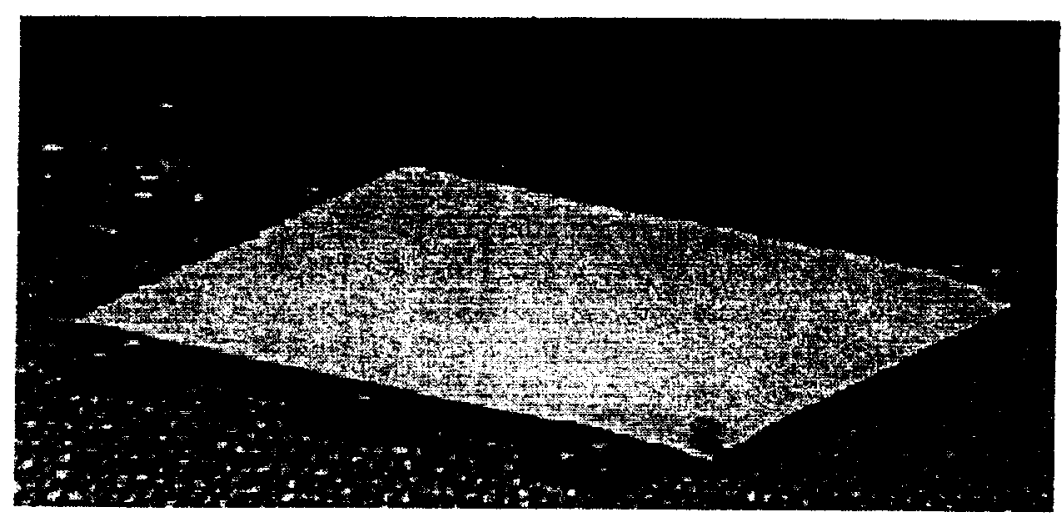

FIGURE 2. ElectroStatic Radiator (Active area is nominally $90 \times 100 \mathrm{~mm}$ )

The second technology, the electrostatic radiator (ESR), is being developed by Sensortex, Inc. located in Kennett Square, Pennsylvania. This technology addresses the active control of thermal radiation with a new and novel approach that eliminates the cost and complexity of existing methods. The ESR uses the fact that a very high emissivity surface, $(\varepsilon \sim 1)$, in good thermal contact with a low emissivity surface, will reach the same temperature. When this occurs, the apparent emissivity of the system (skin plus cover) approaches 1 . However, if the thermal contact is poor, the front surface with the high emissivity film can only radiate the energy it absorbs. In equilibrium, the cover will be at a lower temperature since the absorbed energy, controlling its temperature, comes from a low emissivity surface on the base surface. The amount of energy radiated by the system (cover + skin) is now lower. Thus by switching between these states of good and poor contact between the high emissivity film and the spacecraft skin, the radiation from the skin (which can be treated as an effective emissivity) can be controlled over very wide limits.

To operate properly, the ESR must be capable of achieving intimate thermal contact between the skin and the outer surface. This contact is established by an electrostatic (capacitance-like) hold-down so that the system, consisting of the low emissivity surface with the high emissivity cover, will have a high emissivity when a voltage is applied. Alternately, with the voltage removed, this cover can relax or spring away, thus destroying this thermal contact and resulting in a lowered effective emissivity. When attracted, it makes good conductive thermal contact so the emitting surface of the cover is at the skin temperature. The outer surface of the cover has a high emissivity, so the skin has a high effective emissivity $(\sim 1)$. In the released state, the heat transfer mechanism is now only by radiation, so the only energy that the outer cover can radiate (at equilibrium) is that radiated from the skin, which is fabricated with a low emissivity. The emissivity of the outer skin doesn't change, however its temperature drops and the result is a drop in the radiated energy.

Control of the ESR has the characteristics of low power consumption and simple control circuitry. ESR films have been demonstrated to operate effectively with moderate levels of applied DC voltages (i.e. 200-500V). Application of the film control voltage results in the high emissivity state. Removal of (i.e. shorting) the film control voltage gives the low $\varepsilon$ state. It should be noted that the ESR device basically operates as a high quality capacitor (C $\sim 400$ $\mathrm{pfd} / \mathrm{cm}^{2}$ ). If the control voltage is simply removed (as opposed to shorted) from the ESR film, the high emissivity state will remain for a limited time. Experimental measurements have been conducted to measure the power radiated 
to a cold background in a vacuum environment. GSFC have measured changes in effective emissivity as high as 0.3 while Sensortex have measured changes as high as 0.7 with and without applied voltage. However the planned device for the ST-5 will likely settle at the median of approximately 0.5 .
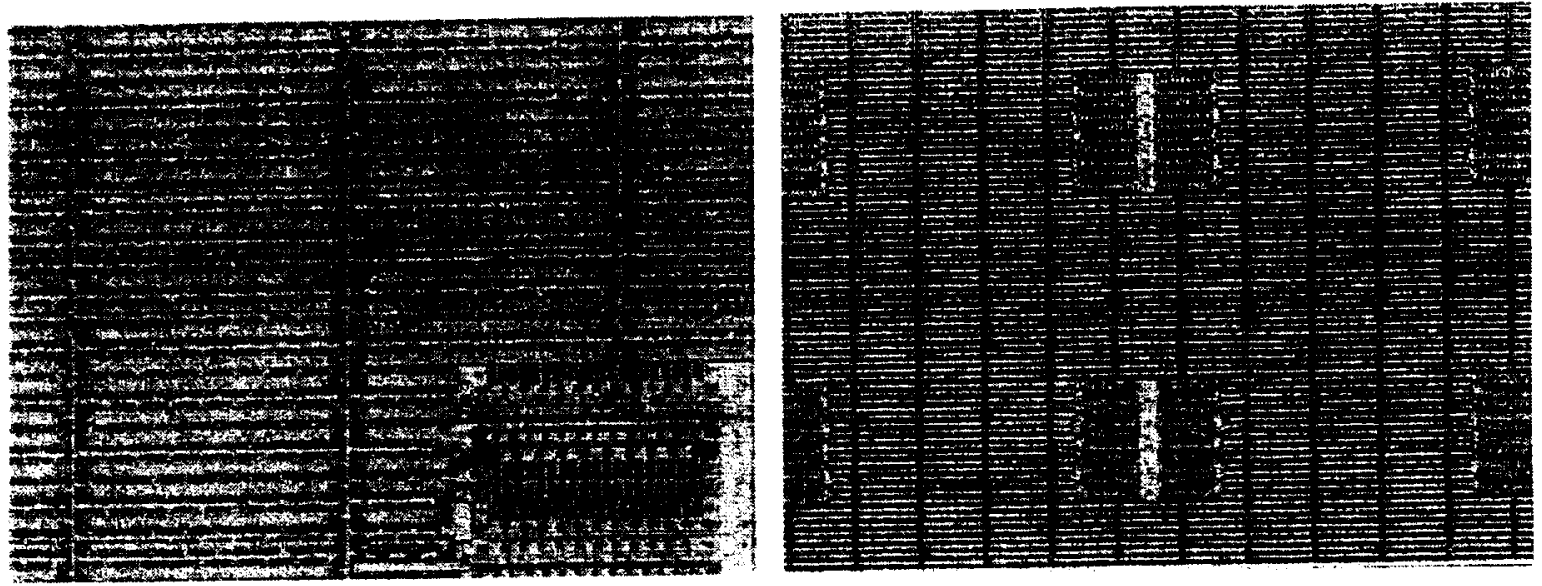

FIGURE 3. MEMS Shutter Array in Different Magnifications.

The third concept, which has been developed at the Johns Hopkins University Applied Physics Laboratory (Champion, 1999; 2001; Garrison, 2000a; 2000b) involves the use of large arrays of micro-machined (MEMS) louvers or shutters to control the radiator emissivity. The most recent design that will be flown on ST-5 is a shutter array actuated by electrostatic comb drives and is fabricated with Sandia National Laboratory's 5-level polysilicon surface machining process, Sandia Ultra-Planner Multi-Level MEMS Technology 5 (SUMMiT V). The design that will be flown on ST-5 is a shutter array based on the use of an electrostatic comb drive. Figure 3 shows two images with different magnification of the array and the comb drive. The base material for the current device is silicon while the shutter is coated with a metal such as gold, which has an absorptivity of 0.3 and a very low emissivity of 0.02 . The total array area on one chip is $5278 \mu \mathrm{m} \times 2632 \mu \mathrm{m}$, with each individual actuator area $225 \mu \mathrm{m} \times 183 \mu \mathrm{m}$ and each group of six actuator elements and shutters $1767 \mu \mathrm{m} \times 876 \mu \mathrm{m}$. Each prototype die contains nine of these groups, which are individually addressable. The ST5 radiator will be composed of about 90 die, each $1 \mathrm{~cm} 2$ in size containing about 60 groups of shutters. The "effective emissivity" of the surface could be modulated in a controlled fashion by varying the total number of arrays that are open. It would also be possible to selectively vary the "effective emissivity" of certain areas of a radiator by modulating those micro-shutters in that area. Emissivity measurements of working devices showed a change in effective emissivity from 0.5 to 0.88 , with the offset of 0.5 given mainly by the inactive area.

\section{INFUSION}

The variable emittance coatings (VEC) will be flown as "self-contained" flight experiments, with two technologies per spacecraft. The basic design of the VEC system consists of the following components and functionality.

\section{VEC Radiator Panel}

The VEC radiator panel is an assembly of multiple VEC devices mounted to an aluminum panel, which in turn, is mounted in a largely thermally isolated fashion to the spacecraft exterior deck surface. The isolation is sized to provide sufficient energy to measure VEC performance, but not enough to seriously affect spacecraft performance in the event of a VEC failure. Each technology has been allocated a volume of $9 \mathrm{~cm} \times 10 \mathrm{~cm} \times 2 \mathrm{~cm}$ and a mass of 200 grams. Performance of these coatings will be measured by temperature data collected from the thermistors mounted at various points on the radiators, as well as analog channels that record voltage and current. The data is collected in the VEC electronics and recorded in the command data and handling system as a function of standard 
housekeeping routines. In addition, two thermistors, which are part of the spacecraft sensors, monitor the VEC temperature and supply that data directly to the onboard computer for health and safety checking even when the VEC is off.

\section{VEC Electronics Unit}

The VEC control electronics, which are housed inside the spacecraft, have been allocated a mass of 50 grams and a maximum of $365 \mathrm{~mW}$ for power. The unit is designed to sample 8 temperature sensors and applies the appropriate voltage to modulate the effective emittance of the variable emittance coating surface. Each VEC technology operates in both a manual and automatic (i.e. set point control) configuration. Selection of the operating mode is commandable from the ground, however, the manual mode, low emittance state is the default mode of operation.

\section{VALIDATION}

Each of the three technology concepts is being developed in parallel through a variety of NASA and DOD funding mechanisms. All three variable emittance technologies have demonstrated adequate performance (a variation in effective emissivity of approximately 0.4 ) in a lab environment, but longevity in the hard vacuum, radiation/atomic oxygen, contamination, thermal cycling, and micrometeoroid environment of space is a real concern that needs to be proven. As such the coatings will undergo extensive environmental testing on the ground. The next step will be to fly the coatings on ST-5 for flight validation. Flight validation of variable emittance techniques will enable future small satellites to be flown with smart, efficient, thermal control skins.

\section{Ground Validation}

As outlined in Table 3 Environmental Test Levels, each technology experiment shall be subjected to full range of testing with appropriate test levels. In addition to extended life cycle testing, exhaustive environmental testing program will include performance measurements (both effective IR emissivity and solar absorptivity), cycling in a vacuum (over 1000 times), exposure to a simulated space environment in GSFC's Solar Wind and UV facilities, and vibration testing. These environmental tests will be followed by another set of performance tests to verify survival.

TABLE 3. Environmental Test Levels.

\begin{tabular}{ll}
\hline Test & \multicolumn{1}{c}{ Conditions } \\
\hline Random Vibration & Flight (Limit) Level $+3 \mathrm{~dB}$, Flight Duration/axis; 3 axes \\
Sinusoidal Vibration & $1.25 \times$ Flight (Limit) Level, Flight Duration/axis; 3 axes 4 oct/min \\
Acoustics & Flight (Limit) Level $+3 \mathrm{~dB}$, Flight Duration \\
Structural Loads & Test: $1.25 \times$ Flight (Limit) Loads, Analysis: $1.4 \times$ Flight (Limit) Loads \\
Mechanical Shock & Analysis: $1.4 \times$ Flight (Limit) Level \\
Thermal Cycle/Vacuum & -60 to $+60^{\circ} \mathrm{C}, 4$ cycles (min), 1 $10^{-5}$ Tort \\
Burn In & $100 \%\left(125^{\circ} \mathrm{C}, 160\right.$ hours min) \\
\hline
\end{tabular}

Flight Validation

Dedicated "Validation Runs" will be performed on each VAR-E technology. As degradation due to space environmental effects is expected to be the major issue for variable emittance coatings, testing over longer periods of time is more important than number of cycles. In order to determine the affect of the space environment, the variable emittance coatings will be operated both at the start of the mission and again towards the end of the mission. In addition, the technologies must operate no less than a total of 100 hours each. Each validation run will 
last at least 2 hours, with a goal of operating continuously over two complete orbits per week. Flight performance measurements will be based on the following:

- Ability to operate in both manual and autonomous modes

- Temperature Telemetry

- Analog measurements of voltage and current to drive the controller and actuate the devices

\section{CONCLUDING REMARKS}

The small sizes of next generation spacecraft, such as those for ST-5, will require new thermal control techniques. The variable emittance coatings technology presented in this paper provide a solution well suited for very small spacecraft. The development of three potentially breakthrough concepts, which include MEMS shutters, electrochromic surfaces and electrostatic devices, were described. At the breadboard stage, these devices have demonstrated the ability to modulate the emissivity of a radiator and are now ready for further development to flight-qualified technology.

\section{NOMENCLATURE}

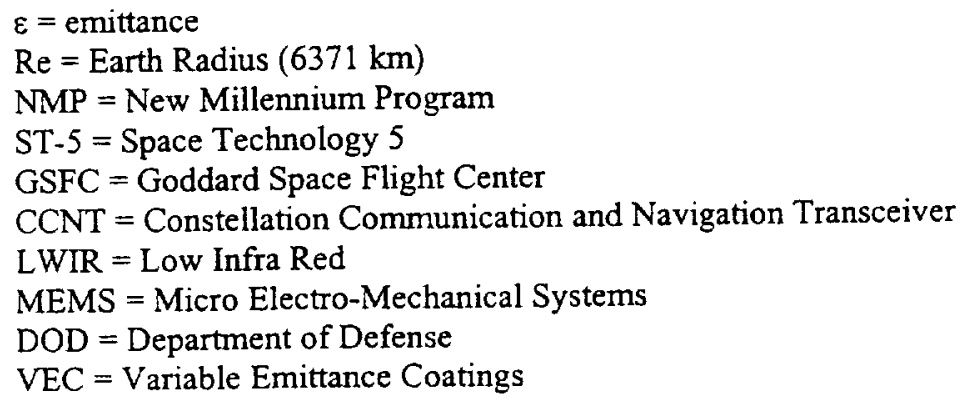

\section{ACKNOWLEDGMENTS}

GSFC is the principal investigator of the Variable Emittance Thermal Suite, which are being developed by Johns Hopkins University Applied Physics Laboratory, Ashwin-Ushas Corporation, Inc., and Sensortex, Inc, is the principal investigator of the Variable Emittance Thermal Suite. Likewise GSFC is the principal investigator of the micro-thruster, which is being developed by UNM-Microelectronics Research Center.

\section{REFERENCES}

Champion, J. L., Osiander, R., Garrison Darrin, M. A., and Swanson, T. D., "MEMS Louvers for Thermal Control," MNT99 Proceedings, 1999, pp. 233-24.

Douglas, D., Michalek, T. and Swanson, T. D., "Design of the Thermal Control System for the Space Technology 5 Microsatellite," $3 I^{\text {st }}$ International Conference on Environmental Systems 2000-01-2214, 2001.

Garrison Darrin, M.A., Osiander, R., Champion, J. L., Swanson, T.D., Douglas, D., and Grob, L. M., "Variable Emissivity Through MEMS Technology," in proceedings of Space Technology and Applications International Forum (STAIF-2000), edited by M. El-Genk, AIP Conference Proceedings 504, New York, 2000, pp. 947-961.

Garrison Darrin, M.A., Osiander, R., Champion, J. L., Swanson, T.D., and Douglas, D., "Variable Emissivity Through MEMS Technology," in proceedings of The Seventh Intersociety Conference on Thermal and Thermomechanical Phenomena in Electronic Systems, edited by G. B. Kromann, J. R. Culham, and K. Ramakrishna, Piscataway, 2000, pp. $215,2000$. 
Osiander, R, Champion, J. L., Garrison Darrin, M. A., Sniegowski, J. J., Rodgers, S. M., Douglas, D., and Swanson, T. D., "Micromachined Louver Arrays for Spacecraft Thermal Control Radiators," American Institule of Aeronautics and Astronautics, Inc., AlAA-2001-0215, 2001. 